\title{
A criança como unidade de cuidado e campo de investigação da enfermagem
}

\section{Child as care unit and area of nursing investigation}

Juliana Freitas Marques ${ }^{1}$, Hete Águida dos Santos ${ }^{1}$

\section{RESUMO}

Nas últimas décadas, a enfermagem tem se destacado no cuidado à criança, exigindo dos profissionais maior capacitação para o enfrentamento desta realidade, o que requer desenvolvimento de pesquisas na área, permitindo aproximação do dia-a-dia vivenciado. Com a perspectiva mencionada, - objetivo desta pesquisa foi investigar as publicações de enfermagem acerca do cuidado à criança nos anos de 2005 a 2010. Trata-se de um estudo de revisão bibliográfica realizado entre fevereiro e abril de 2011. Foi utilizada a base de dados SciELO e procuradas publicações com as palavras-chave "enfermagem", "cuidado" e "criança". Incluíram-se publicações de periódicos de enfermagem nos anos de 2005 a 2010 e excluídas as que não tinham o cuidado à criança como foco temático. Após o refinamento, foram selecionados 49 artigos. A maior quantidade de estudos publicados foi no ano de 2010, totalizando 13 artigos. A Revista da Escola Anna Nery apresentou maior número de publicações, totalizando 12 artigos. Trinta e dois estudos utilizaram a abordagem qualitativa e 33 não apresentaram referencial teórico. A análise dos textos possibilitou traçar um panorama temático, procurando subsidiar as pesquisas futuras sobre enfermagem pediátrica e retomando o sentido de considerar a criança um dos principais focos de atuação do cuidado da enfermagem.

Palavras-chave: enfermagem pediátrica; cuidado da criança; revisão.

\begin{abstract}
In recent decades, nursing has been prominent in child care requiring more training of professionals to confront this reality, which requires further research in the area, allowing approximation to the reality experienced. From this perspective, the objective of this study was to investigate nursing publications about child care in the years 2005 to 2010. It is a bibliographic review carried out between February and April of 2011. We used the SciELO database and the publications were searched with the keywords "nursing", "care" and "child". Publications of nursing periodicals in the years 2005 to 2010 were included and texts that did not have child care as a thematic focus were excluded. After refinement, 49 articles were selected. The largest number of studies was published in 2010, totaling 13. The Revista da Escola Anna Nery presented the highest number of publications (12). Thirty two studies used a qualitative approach and 33 did not use the theoretical reference. The analysis of texts allowed to thematic outlook, seeking to subsidize future research on pediatric nursing and taking up the sense of considering the child as a major focus of nursing care activities.
\end{abstract}

Keywords: pediatric nursing; child care; review. 


\section{INTRODUÇÃO}

No contexto brasileiro, a atenção à saúde da criança vem sendo discutida durante os últimos anos. O início da década de 1990 foi o grande propulsor disso, por meio da criação de programas específicos e do Estatuto da Criança e do Adolescente (ECA), que garante os direitos legais a esse grupo antes desconsiderado.

Com o ECA, promulgado pela Lei $n^{\circ} 8.069$, de 13 de julho de 1990, a criança passa a gozar de direitos fundamentais, sendo garantidos a saúde, alimentação, proteção, educação, esporte, lazer e cultura. E, além da família, o Estado como um todo possui o dever de zelar e garantir esses direitos básicos ${ }^{1}$.

Tais avanços na atenção à criança não ocorreram somente no Brasil. Mundialmente houve a criação de programas e órgãos não governamentais que dedicam o seu trabalho ao bem-estar e saúde da população em geral, incluindo a criança e o adolescente, como o Fundo das Nações Unidas para a Infância (UNICEF), a Organização Mundial de Saúde (OMS) e a Organização Pan-Americana de Saúde (OPAS) ${ }^{2}$.

Atrelado ao avanço das políticas públicas, houve aumento de ações de saúde, como o crescimento da cobertura vacinal, melhores condições de saneamento básico e nutrição, a introdução e expansão da terapia de reidratação oral e a criação de programas de controle da diarreia e de incentivo ao aleitamento mater$\mathrm{no}^{3}$. Entretanto, apesar dos índices de mortalidade infantil terem reduzido no país, ainda se observa que as principais causas de morte na infância estão ligadas às condições socioeconômicas e dificuldades de acesso aos serviços de saúde, ou seja, situações passíveis de serem evitadas ${ }^{4}$.

Em estudo recente, as principais causas de hospitalização no Brasil entre crianças menores de quatro anos foram as doenças do aparelho respiratório, seguidas das doenças parasitárias e infecciosas, predominantes nas regiões Norte e Nordeste. Dessa forma, as políticas de saúde devem ser coerentes com cada realidade, tanto no que diz respeito à distribuição de recursos quanto na adoção de estratégias para a melhoria considerando a população infantil ${ }^{5}$.

Diante dos indicadores de saúde, o cuidado ofertado à criança torna-se decisivo e tem impacto duradouro sobre o desenvolvimento e o crescimento, o que promove qualidade de vida e melhora nos índices epidemiológicos. Entretanto, atenta-se que o cuidado deve ser imbuído de atenção, capaz de gerar confiança e vínculo afetivo entre a criança e o profissional de saúde ${ }^{6}$.

Envolvidos neste processo encontram-se os profissionais de enfermagem que atuam diretamente nos cuidados oferecidos à criança e sua família. A criança é foco de trabalho das práticas assistenciais e de pesquisas na área, sendo abordada em seu contexto de cuidado, por meio de ações clínico-preventivas como também intervencionistas.

É importante salientar que o cuidado de enfermagem à criança não se restringe à busca do êxito técnico, pois apesar da preocupação do enfermeiro com as práticas de saúde por meio de ações curativistas, deve-se atentar para as atitudes compreensivas no processo de cuidar, caminhando para a construção de novos entendimentos das situações que a criança e a família enfrentam ${ }^{7}$.

Com essa perspectiva, o cuidado de enfermagem à criança implica em ações curativas, preventivas e de promoção da saúde, indissociáveis entre si, construindo espaços de cuidado no contexto familiar e social. Assim, a enfermagem em saúde da criança deve articular o êxito técnico à longitudinalidade do cuidado, buscando, no acompanhamento de crescimento e desenvolvimento, intervenções que estejam voltadas para os bons indicadores de saúde ${ }^{7}$.

A proposta de inclusão de novas ações no cuidado à criança parte, muitas vezes, da realização de pesquisas nas quais são comprovadas por meio de métodos, técnicas e teorias utilizadas pela enfermagem. As experiências científicas no trabalho com a criança encontram-se registradas em teses, dissertações, livros e artigos da área. Dessa forma, destacam-se as inúmeras possibilidades de reconhecer a importância da criança no contexto atual da saúde. Entende-se que estudos sobre o cuidado relacionado a este público podem contribuir para o planejamento das intervenções e a melhoria do cuidado de enfermagem.

Considerando o contexto do estudo que permeia o cuidado do enfermeiro à criança, torna-se importante conhecer o que a enfermagem brasileira vem pesquisando e publicando nos últimos anos, verificando, assim, as práticas profissionais mais cotidianas. Com isso, o presente estudo pretende reforçar e ampliar a reflexão da enfermagem ao aprimoramento do cuidar à criança com o objetivo de analisar a produção do conhecimento sobre o assunto no período de 2005 a 2010.

\section{MÉTODO}

Trata-se de uma pesquisa bibliográfica, não sistematizada, que buscou conhecer os estudos sobre o tema "cuidado à criança", permitindo caracterizar e analisar as tendências da produção científica na área da enfermagem.

O levantamento foi realizado entre os meses de fevereiro e abril de 2011 utilizando as palavras-chave "enfermagem", "criança" e "cuidado". Para a coleta de dados, levou-se em conta a base de dados Scientific Electronic Library Online (SciELO), uma biblioteca piloto de fácil e gratuito acesso na internet, que abrange uma coleção selecionada de periódicos científicos brasileiros.

$\mathrm{Na}$ primeira etapa da pesquisa foram coletadas 157 publicações. Os critérios de inclusão utilizados foram: estudos publicados em periódicos de enfermagem no período de 2005 a 2010, em língua portuguesa, na forma de texto completo. Excluíram-se as que não tinham o cuidado de enfermagem à criança como foco temático.

Com o refinamento, foram selecionadas 49 publicações que contemplaram o corpus da revisão bibliográfica (Tabela 1).

Após a leitura dos textos selecionados, organizou-se o material a partir do banco de dados, denominado roteiro de coleta de dados, que continha as variáveis "tipo e natureza do estudo", "ano de 
publicação", "periódico de publicação", "utilização de referencial teórico" e "área temática".

Os dados foram apresentados em tabelas, e as variáveis analisadas de forma descritiva com medidas de frequência absoluta simples e percentual. A análise e discussão das temáticas levantadas ocorreram a partir da interpretação das leituras e releituras dos textos selecionados. Tem-se a clareza de que esta pesquisa não contempla todas as publicações brasileiras de enfermagem sobre o assunto. Contudo, a análise dos textos possibilitou traçar um panorama temático, procurando subsidiar as pesquisas futuras sobre o cuidado de enfermagem e o cuidado à criança.

\section{RESULTADOS E DISCUSSÃO}

Dos 49 artigos selecionados, a maioria (13) foi publicada em 2010. Na sequência aparecem os anos 2007 (10), 2009 (nove), 2005 (seis), 2008 (seis) e 2006 (cinco). Relacionou-se ainda o ano de publicação com os periódicos encontrados. Tem-se a Revista da Escola Anna Nery de Enfermagem com maior número de artigos (12) sobre a temática. Em seguida está a Revista Latino-Americana de Enfermagem (dez), Acta Paulista de Enfermagem (oito), Revista Brasileira de Enfermagem (sete), Revista da Escola de Enfermagem da USP (quatro), Revista Gaúcha de Enfermagem (quatro) e Texto \& Contexto Enfermagem (quatro) (Tabela 1).

Foram analisadas também as publicações segundo a natureza do estudo. Dentre os artigos selecionados, observou-se que a maioria (32) foi de natureza qualitativa. Duas publicações utilizaram a abordagem quantiqualitativa ou multimétodos e três abordaram a natureza quantitativa. Além disso, sete estudos eram do tipo revisão bibliográfica, três relatos de experiência e dois estudos de reflexão teórica (Tabela 2).
Em relação à enfermagem, observa-se que a natureza qualitativa vem se destacando na maioria dos estudos desenvolvidos, o que, na área da saúde da criança, não poderia ser diferente. A opção por essa abordagem permite a descrição das experiências humanas tal como é vivida e definida por seus próprios atores. Os pesquisadores da abordagem coletam e analisam material pouco estruturado que propicia campo livre ao rico potencial das percepções e subjetividades dos seres humanos. Com isso, a enfermagem em saúde da criança busca, a partir da natureza qualitativa, descrever e compreender a realidade com as experiências dos sujeitos. O objeto de estudo não é reduzido a variáveis e sim à complexidade e totalidade de seu contexto bem como às práticas e interações da vida cotidiana ${ }^{8,9}$.

Em alguns artigos, destaca-se o uso do referencial teórico, que norteia a maneira pela qual o autor descreve e busca apreender questões sobre o estudo em construção, recorrendo a teorias existentes sobre a área de pesquisa e promovendo a sustentação da ideia que defende.

Verificou-se que dentre as publicações selecionadas, 33 não fizeram uso do referencial teórico e somente 16 recorreram ao recurso (Tabela 3 ).

Os modelos teóricos nos estudos sobre a criança na enfermagem apresentam alguns caminhos que ajudam os profissionais que trabalham com esses pacientes a pensar sobre a melhor intervenção e o processo de cuidado, necessário para reverter ou amenizar situações de conflitos que interferem no processo de saúde e doença. Dentre as teorias que nortearam os estudos, destacam-se: Teoria Humanística de Paterson e Zderard, Estudos Fenomenológicos, Interacionismo Simbólico, Teoria da Adaptação de Calista Roy, Teoria do Apego, Corrente Histórico-social, Estudos Culturais e Modelo Calgary de Avaliação e Intervenção Familiar.

Tabela 1: Características dos estudos incluídos na revisão bibliográfica da produção científica da enfermagem no cuidado à criança, base SciELO, anos 2005 a 2010 - Arapiraca (AL)

\begin{tabular}{|c|c|c|c|c|c|c|c|}
\hline Periódico & 2005 & 2006 & 2007 & 2008 & 2009 & 2010 & Autores \\
\hline $\begin{array}{l}\text { Acta Paulista de } \\
\text { Enfermagem }\end{array}$ & 0 & 0 & 2 & 2 & 3 & 1 & $\begin{array}{l}\text { Rossi CS, Rodrigues BMRD; Cunha PJ, Zagonel IPS; Medeiros G, et } \\
\text { al.; Monteiro FPM, et al.; Rocha DLB, Zagonel IPS; Guiller CA, Dupas } \\
\text { G, Pettengill MAM; Parisi TCH, Coelho ERB, Malleiro MM; Oliveira VC, } \\
\text { Cadette MMM. }\end{array}$ \\
\hline $\begin{array}{l}\text { Escola Anna Nery } \\
\text { Enfermagem }\end{array}$ & 0 & 1 & 2 & 2 & 2 & 5 & $\begin{array}{l}\text { Pedroso MLR, Motta MGC; Monteiro FPM, Caetano JA, Araújo TL; Coelho } \\
\text { ACO, et al.; Paula CC, Crossetti MGO; Christoffel MM, Pacheco STA, Reis } \\
\text { CSC; Avanci BS, et al.; Carneiro DMS, Souza IEO, Paula CC; Woiski ROS, } \\
\text { Rocha DLB; Souza TV, Oliveira ICS; Nascimento LC, et al.; Leite NSL, } \\
\text { Cunha SR; Vieira M, Padilha MICS. }\end{array}$ \\
\hline $\begin{array}{l}\text { Revista da Escola de } \\
\text { Enfermagem USP }\end{array}$ & 0 & 0 & 0 & 2 & 2 & 0 & $\begin{array}{l}\text { Klassmann J, et al.; Pimenta EAG, Collet N; Leite TMC, Shimo AKK; } \\
\text { Lima RAG, et al. }\end{array}$ \\
\hline $\begin{array}{l}\text { Revista Brasileira de } \\
\text { Enfermagem }\end{array}$ & 3 & 1 & 2 & 0 & 0 & 1 & $\begin{array}{l}\text { Lemos LMD, et al.; Regis FC, Kakehashi YT, Pinheiro EM; Simpionato } \\
\text { E, Correia CC, Rocha SMM; Queiroz FC, et al.; Ravelli APX, Motta MGC; } \\
\text { Ferreira CCM, Remedi PP, Lima RAGL; Beck ARM, Lopes MHBM. }\end{array}$ \\
\hline $\begin{array}{l}\text { Revista Gaúcha de } \\
\text { Enfermagem }\end{array}$ & 0 & 0 & 0 & 0 & 0 & 4 & $\begin{array}{l}\text { Pedroso MLR, Motta MGC; Jansen MF, Santos RM, Favero L; Quirino DD, } \\
\text { Collet N, Neves AFGB; Costa TF, Ceolim MF. }\end{array}$ \\
\hline $\begin{array}{l}\text { Revista } \\
\text { Latino-Americana de } \\
\text { Enfermagem }\end{array}$ & 2 & 3 & 3 & 0 & 1 & 1 & $\begin{array}{l}\text { Costa TF, Ceolim MF; Nascimento LC, et al.; Mello DF, Lima RAG; } \\
\text { Pizzignacco TMP, Lima RAG; Flores Peña, et al.; Silveira AO, Angelo M; } \\
\text { Pinto JP, Barbosa VL; Ortiz MCA, Lima RAG; Costa JC, Lima RAG; } \\
\text { Sabatés AL, Borba RIH; Pedro ICS, et al.. }\end{array}$ \\
\hline $\begin{array}{l}\text { Texto \& Contexto } \\
\text { Enfermagem }\end{array}$ & 1 & 0 & 1 & 0 & 1 & 1 & $\begin{array}{l}\text { Yamamoto DM, et al.; Paula CC, Crossetti MGO; Freitas HMB, et al.; } \\
\text { Ribeiro RLR, Rocha SMM. }\end{array}$ \\
\hline TOTAL & 6 & 5 & 10 & 6 & 9 & 13 & - \\
\hline
\end{tabular}


Ressalta-se que foram poucas as pesquisas embasadas em um referencial teórico, fato que prejudica a cientificidade da enfermagem, pois são as teorias que permitem maior embasamento e aprofundamento dos estudos na área.

Outro aspecto relevante contemplado neste estudo é a identificação das áreas temáticas utilizadas nas produções científicas da enfermagem no cuidado à criança, compreendidas no período de 2005 a 2010. Nesse sentido, torna-se importante conhecer as principais áreas enfocadas, podendo servir, assim, como instrumento de análise para profissionais de enfermagem e áreas afins que visem conhecer e desenvolver novos estudos na área (Tabela 4).

$\mathrm{Na}$ análise, constatou-se um quantitativo de dez artigos referentes ao cuidado à criança hospitalizada e sua família, nos quais pode ser evidenciada a preocupação com a busca pelo melhor aperfeiçoamento da assistência.

Tabela 2: Natureza dos estudos empregados nas produções científicas da enfermagem no cuidado à criança, na base SciELO, anos 2005 a 2010 - Arapiraca (AL)

\begin{tabular}{lcc}
\hline Natureza do estudo & $\mathbf{n}$ & $\%$ \\
\hline Qualitativo & 32 & 65,3 \\
Quantiqualitativo & 2 & 4,1 \\
Quantitativo & 3 & 6,1 \\
Revisão bibliográfica & 7 & 14,3 \\
Relato de experiência & 3 & 6,1 \\
Reflexão teórica & 2 & 4,1 \\
Total & 49 & 100,0
\end{tabular}

Tabela 3: Uso de referencial teórico em produções científicas da enfermagem no cuidado à criança, na base SciELO, nos 2005 a 2010 - Arapiraca (AL)

\begin{tabular}{lcc}
\hline Referencial teórico & $\mathbf{n}$ & $\%$ \\
\hline Não & 33 & 67,3 \\
Sim & 16 & 32,7 \\
Total & 49 & 100,0 \\
\hline
\end{tabular}

Fonte: SciELO

Tabela 4: Temáticas principais das produções científicas da enfermagem no cuidado à criança, na base SciELO, nos 2005 a 2010 - Arapiraca (AL)

\begin{tabular}{|c|c|c|}
\hline Área de concentração & $\mathbf{n}$ & $\%$ \\
\hline $\begin{array}{l}\text { Cuidado da criança hospitalizada e } \\
\text { sua família }\end{array}$ & 10 & 20,4 \\
\hline Lúdico como estratégia de cuidado & 9 & 18,3 \\
\hline Cuidado da criança com câncer & 5 & 10,2 \\
\hline Cuidado da criança com HIV/AIDS & 4 & 8,2 \\
\hline $\begin{array}{l}\text { Sistematização da assistência de } \\
\text { enfermagem }\end{array}$ & 4 & 8,2 \\
\hline $\begin{array}{l}\text { Processo de trabalho de } \\
\text { enfermeiro }\end{array}$ & 2 & 4,1 \\
\hline $\begin{array}{l}\text { Vínculo mãe e filho/método } \\
\text { mãe-canguru }\end{array}$ & 2 & 4,1 \\
\hline $\begin{array}{l}\text { Cuidado de enfermagem no } \\
\text { pós-operatório }\end{array}$ & 2 & 4,1 \\
\hline Outros & 11 & 22,4 \\
\hline Total & 49 & 100,0 \\
\hline
\end{tabular}

Fonte: SciELO
O que se pode apreender das publicações supracitadas é que a inserção de um familiar durante o processo de hospitalização infantil se revela muito importante, já que a criança estará em ambiente que não lhe é própria, no qual passará por rotinas que certamente causarão desconforto e estresse. Tal situação pode ser amenizada com a presença de algum familiar, mas ao lado desse benefício tem-se outra preocupação para equipe de saúde, pois agora não apenas a criança, mas o binômio criança-família merece cuidado e atenção.

Dessa forma, a inclusão de parentes no ambiente de cuidado hospitalar requer modificação no cotidiano do trabalho do enfermeiro. Nesta perspectiva, o relacionamento entre a equipe de enfermagem e a família deve ser dinâmico, havendo cooperação entre todos os atores envolvidos para que o cuidado seja efetivado de forma contínua e integral. A integração entre os profissionais e os parentes permite que estes continuem prestando os cuidados no domicio ${ }^{10}$.

O vínculo entre mãe e filho é uma temática estudada há mais de meio século, e os estudos constatam que o afastamento abrupto de ambos pode ocasionar sérios danos à criança e sua saúde ${ }^{11}$. Com a regulamentação do Estatuto da Criança e do Adolescente em 1990, o Brasil avançou em relação à humanização da assistência à criança, e a permanência dos pais ou responsáveis foi legalizada em casos de internação, devendo os serviços de saúde oferecer condições adequadas para a presença dos pais em tempo integral $^{12}$.

A segunda área de concentração com o maior número de publicações (nove) refere-se ao lúdico como estratégia do cuidado de enfermagem. Os estudos relatam a utilização do brinquedo terapêutico, da música e do teatro como instrumentos que amenizam as tensões e facilitam o enfrentamento de situações estressantes, como as vivenciadas durante a hospitalização. Percebe-se também que a enfermagem tem grande parcela de contribuição para que essa tensão seja minimizada, pois os profissionais abordam de modo a respeitar a criança em sua individualidade e nisso consiste o sucesso de sua recuperação.

O lúdico tem um importante valor terapêutico: a utilização de brinquedos, músicas e dramatizações assegura o cuidado integral e humanizado. Para a enfermagem, essas estratégias permitem mais interação entre o profissional e a criança; o cuidar, visto como a dicotomia entre arte e ciência, pode ser expresso de modo sensível e criativo, proporcionando o crescimento e desenvolvimento do paciente ${ }^{13}$.

Outra área relevante que surgiu durante a análise das publicações foi o cuidado à criança com câncer. Os estudos enfocaram os desafios encontrados pela enfermagem quanto à abordagem à pediatria oncológica, pois as dificuldades transcendem a vontade de saber e conhecer mais sobre o assunto, já que se trata de um obstáculo encontrado também durante a graduação dos profissionais.

Percebe-se que existe uma lacuna considerável na capacitação da enfermagem em oncologia pediátrica, pois a maioria 
dos cursos universitários geralmente não oferece aprofundamento nessa área. Vale salientar que as Diretrizes Curriculares Nacionais para os Cursos de Graduação em Enfermagem pregam a formação do enfermeiro generalista. Todavia, o perfil epidemiológico nacional e mundial deve ser observado e as devidas adaptações curriculares feitas para que a formação atenda às realidades específicas $^{14-16}$

O cuidado dispensado ao paciente com AIDS evidenciou, por meio dos estudos, os desafios que os cuidadores de crianças nessa situação encontram, pois é necessária mais sensibilização dos profissionais e da família para que seja considerada não um indivíduo doente, mas um sujeito único que possui ilimitadas possibilidades de viver com saúde, independente de idade, sexo e/ou condição social ${ }^{17}$.

Assim, a compreensão do significado ou do sentimento de uma criança com HIV/AIDS possibilita uma experiência que se expressa de forma singular, integrando, dessa forma, o processo de adoecimento. Contudo, o cuidado de enfermagem precisa ser abrangente e contextualizado; precisa levar em conta que a criança é um ser único e complexo e que convive com o contrassenso de estar viva e, ao mesmo tempo, leva em conta as instabilidades do momento, assim como as incertezas do futuro ${ }^{17}$.

A Sistematização da Assistência de Enfermagem (SAE) no cuidado à criança surgiu nos estudos elencados por meio de pesquisas e relatos de experiências, demonstrando a relação entre o planejamento do cuidado e sua contribuição para a promoção da saúde da criança. Percebeu-se a importância das etapas do processo de enfermagem na condução de um cuidado efetivo, destacando-as como importante fator no processo da relação terapêutica entre profissional, criança e família, de forma a garantir a consolidação das informações e orientações prestadas ${ }^{18}$.

A temática "Processo de trabalho do enfermeiro" surgiu em duas publicações selecionadas. Destaca-se que as mudanças ocorridas no cuidado à criança hospitalizada, como a presença do acompanhante durante a internação, influenciaram na dinâmica de trabalho do profissional, principalmente pelo maior tempo que precisa dispor para prestar assistência tanto à criança, quanto à família. No entanto, observa-se uma série de conflitos que envolvem o modo de assistir o paciente hospitalizado, em que, na dinâmica das relações, surgem situações complexas, requerendo mudanças na atitude do enfermeiro no cotidiano da prática assistencial ${ }^{10}$.

A Neonatologia, apesar de ser considerada outra área do cuidar em enfermagem, surgiu dentre os estudos pesquisados. Os artigos fazem menção ao Método Mãe-canguru, tecnologia do cuidado relevante, já que interfere diretamente no crescimento e desenvolvimento da futura criança.

Este método surgiu da necessidade de encontrar soluções para a superlotação das unidades neonatais, que muitas vezes mantinham mais de dois recém-nascidos na mesma incubadora ${ }^{19}$. Nos estudos analisados, observou-se a importância da implementação do método para bebês prematuros de baixo peso, pois ajuda tanto no desenvolvimento nutricional do recém-nascido quanto no fortalecimento do vínculo mãe e filho.

As demais áreas de concentração encontradas, tendo a criança como unidade de cuidado, evidenciam as diversas facetas da enfermagem em relação ao cuidado e apoio dispensado à criança em seu contexto, seja ele qual for.

A enfermagem precisa continuar desenvolvendo tecnologias do cuidar que dêem conta das novas demandas que vêm se configurando no campo dos cuidados à saúde. $\mathrm{O}$ aprimoramento das terapias intensivas - a criança dependente de tecnologia -, os transplantes de órgãos e o cuidado domiciliar, bem como a educação em saúde por meio de atividades lúdicas, colocam a enfermagem diante de novas perspectivas e campos de inserção. Além do que, a produção do conhecimento orientada por esses múltiplos objetos de estudo constitui-se em uma necessidade social para fundamentar a prática profissional cientificamente estruturada ${ }^{20}$.

\section{CONCLUSÃO}

Nos últimos anos, a alteração no perfil epidemiológico das doenças prevalentes na infância exige maior capacitação dos profissionais, o que se constitui um desafio para a enfermagem, que deve voltar suas ações para a promoção da saúde prevenção dos agravos e intervenção dos cuidados específicos. A atuação do enfermeiro deve atrelar a assistência direta à criança e sua família com o desenvolvimento de pesquisas na área, conforme as demandas e necessidades exigidas pela população específica.

Nesse sentido, a enfermagem possui inúmeras possibilidades de prestar suas habilidades e competências na saúde infantil. Neste estudo, pôde-se evidenciar que as pesquisas vêm destacando uma dimensão mais humana do cuidar à criança e sua família, fato esse expresso pelo significativo número de artigos qualitativos na área.

Observam-se nos estudos analisados os diversos contextos nos quais a criança e sua família são pesquisadas. No entanto, tornam-se necessárias mais pesquisas, inclusive em outras áreas do conhecimento, no intuito de conhecer e entender as mudanças que ocorrem no contexto da enfermagem pediátrica. É importante, ainda, que sejam desenvolvidos estudos estatísticos com base na epidemiologia local, pois no presente trabalho observou-se carência de pesquisas com essa abordagem.

A divulgação dos resultados desta pesquisa poderá subsidiar novos estudos e direcionar a assistência na área da enfermagem pediátrica, trazendo contribuições para os profissionais de saúde como também para a comunidade acadêmica.

Neste artigo não houve pretensão de exaurir o tema. Logo, é necessária a constante leitura e realização de novas pesquisas sobre o assunto proposto, com o escopo de adaptar-se às novas tendências que são necessárias para aprimorar o foco supracitado. 


\section{REFERÊNCIAS}

1. Brasil. Ministério da Saúde. Estatuto da criança e do adolescente. Brasília: Ministério da Saúde; 1991.

2. Apostólico MR, Cubas MR, Altino DM, Pereira KCM, Egry EY. Contribuição da CIPESC na execução das políticas de atenção à saúde da criança no município de Curitiba, Paraná. Texto Contexto Enferm. 2007;16(3):453-62.

3. Prado SRLA, Fujimori E. Conhecimento materno/familiar sobre o cuidado prestado à criança doente. Rev Bras Enferm. 2006;59(4):492-6.

4. Ministério da Saúde. Secretaria de Atenção à Saúde. Agenda de compromissos para a saúde integral da criança e redução da mortalidade infantil. Brasília: Ministério da Saúde; 2004.

5. Oliveira BRG, Viera CS, Collet N, Lima RAG. Causas de hospitalização no SUS de criancas de zero a quatro anos no Brasil. Rev Bras Epidemiol. 2010;13(2):268-77.

6. Martins J, Veríssimo MLOR, Oliveira MA. Avaliação dos instrumentos do projeto "nossas crianças: janelas de oportunidades", segundo agentes comunitários de saúde. Texto Contexto Enferm. 2008; 17(1):106-14.

7. Mello DF, Lima RAG. Êxito técnico, sucesso prático e sabedoria prática: bases conceituais hermenêuticas para o cuidado de enfermagem à criança. Rev Latino-Am Enferm. 2009;17(4):580-5.

8. Minayo MCS. O desafio do conhecimento: pesquisa qualitativa em saúde. São Paulo: Hucitec; 2006.

9. Flicck U. Uma introdução à pesquisa qualitativa. Porto Alegre: Bookman; 2004.

10. Yamamoto DM, Oliveira BRG, Viera CS, Collet N. O processo de trabalho dos enfermeiros em Unidades de Alojamento Conjunto pediátrico de instituicões hospitalares públicas de ensino do Paraná. Texto Contexto Enferm. 2009;18(2):224-32.

11. Siqueira LS, Sigaud CHS, Rezende MA. Fatores que apoiam e não apoiam a permanência de mães acompanhantes em unidade de pediatria hospitalar. Rev Esc Enferm USP. 2002;36(3):270-5.
12. Faquinello P, Higarashi LH, Marcon SS. O atendimento humanizado em unidade pediátrica: percepção do acompanhante da criança hospitalizada. Texto Contexto Enferm. 2007;16(4):609-16.

13. Ravelli APX, Motta MGC. O lúdico e o desenvolvimento infantil: um enfoque na música e no cuidado de enfermagem. Rev Bras Enferm. 2005:58(5):611-3.

14. Amador DD, Gomes IP, Coutinho SED, Costa TNA, Collet N. Concepção dos enfermeiros acerca da capacitação no cuidado à criança com câncer. Texto Contexto Enferm. 2011;20(1):94-101.

15. Beck ARM, Lopes MHBM. Cuidadores de crianças com câncer: aspectos da vida afetados pela atividade de cuidador. Rev Bras Enferm. 2007;60(6):670-5

16. Ministério da Educação. Conselho Nacional de Educação. Câmara de Educação Superior. Resolução CNE/CES n. 3, de 7 de novembro de 2001. Institui as diretrizes curriculares nacionais do curso de graduação em enfermagem. Diário Oficial da República Federativa da União. Brasília: 9 nov. 2001.

17. Freitas HMB, Backes DS, Pereira ADA, Ferreira CLL, Marchiori MRC, Souza MHT, et al. Significados que os profissionais de enfermagem atribuem ao cuidado de crianças institucionalizadas com aids. Texto Contexto Enferm. 2010;19(3):511-7.

18. Christoffel MM, Pacheco STA, Reis CSC. Modelo Calgary de avaliação da família de recém-nascidos: estratégia pedagógica para alunos de enfermagem. Esc Anna Nery Rev Enferm. 2008;12(1):160-5.

19. Lamy ZC, Gomes MASM, Gianini NOM, Hennig MA. Atenção humanizada ao recém-nascido de baixo peso - método Canguru: a proposta brasileira. Ciênc Saúde Coletiva. 2005;10(3):659-68.

20. Cabral IE. Desafios e perspectivas do cuidar de enfermagem na saúde da criança. Esc Anna Nery. 2009;13(4):691-3.

\section{Endereço para correspondência}

Juliana Freitas Marques - Rua Thereza Auto Teófilo, 56, casa 3 -

Barra Mar - CEP: 57180-000 - Barra de São Miguel (AL), Brasil -

E-mail:julianaf_marques@hotmail.com

Conflito de interesse:nada a declarar. 